\title{
Anisotropic Diffusion of Tensor Fields for Fold Shape Analysis on Surfaces
}

\author{
Maxime Boucher ${ }^{1,2,3}$, Alan Evans ${ }^{2}$, and Kaleem Siddiqi ${ }^{1}$ \\ 1 School of Computer Science and Centre for Intelligent Machines, McGill University. \\ ${ }^{2}$ McConnell Brain Imaging Center, Montreal Neurological Institute, McGill \\ University. \\ ${ }^{3}$ Corresponding Author: boucher@bic.mni.mcgill.ca
}

\begin{abstract}
The folding pattern of the human cortical surface is organized in a coherent set of troughs and ridges, which mark important anatomical demarcations that are similar across subjects. Cortical surface shape is often analyzed in the literature using isotropic diffusion, a strategy that is questionable because many anatomical regions are known to follow the direction of folds. This paper introduces anisotropic diffusion kernels to follow neighboring fold directions on surfaces, extending recent literature on enhancing curve-like patterns in images. A second contribution is to map deformations that affect sulcal length, i.e., are parallel to neighboring folds, with other deformations that affect sulcal length, within the diffusion process. Using the proposed method, we demonstrate anisotropic shape differences of the cortical surface associated with aging in a database of 95 healthy subjects, such as a contraction of the cingulate sulcus, shorter gyri in the temporal lobe and a contraction in the frontal lobe.
\end{abstract}

Keywords: Surface Morphometry, Statistical Shape Analysis

\section{Introduction}

The folding pattern of the human cortical surface results in a coherent set of troughs and ridges, which to some extent are similar across subjects. Folds can mark important anatomical boundaries and they vary in width and length across individuals. Differences in fold shape can be associated with aging, gender, disease and underlying white matter connectivity.

Several methods exist to analyze cortical surface fold shape. For example, one approach is to compute the average shape of a set of surfaces using an iterative registration process, following which the mechanical deformation field required to deform an individual surface onto the mean surface is used as a statistical shape model $[4,9,11,2]$. This approach faces a statistical estimation problem: the effect of a factor on the shape of the underlying cortical surface needs to be estimated in the presence of statistical noise. The presence of noise reduces the confidence that the estimated effect of a factor is different from 
zero and reduces the detection power of the statistical framework. One way to mitigate this problem is to locally average the shape measurements using surface diffusion kernels [4]. However, isotropic diffusion averages measurements across neighboring neuroanatomic regions, which is generally not desirable.

In this paper we propose to use anisotropic diffusion on surfaces to highlight patterns that occur on specific folds, an example of which is shown in Figure 1. We use the approach of surface-based morphometry for statistical analysis, where each individual cortical surface is viewed as a deformed version of a template surface. We use a simplicial mesh as a representation to find the correspondence between vertices on the template surface and each of the individual surfaces via a surface registration process. We then carry out anisotropic diffusion in tensorbased morphometry and statistical analysis on the deformation tensor of that mapping, which is the 2-dimensional version of the one used in [9].

There are two main contributions in this paper. The first is a method to anisotropically diffuse a scalar field on a surface, such that the diffusion speed is faster along neighboring fold orientations, as shown in Figure 1. This differs from other anisotropic diffusion schemes that are designed primarily to suppress diffusion across creases [5]. The second contribution is related to tensor diffusion on surfaces. The added complexity is that tensors have a directional component, which needs to be taken into consideration. We propose to keep tensors aligned with neighboring folds within the diffusion process. This requires us to rotate the tensor field within the diffusion scheme according to how the neighboring folds change in orientation. The method we develop is similar to the one proposed in [8] for enhancing curve like patterns in images.
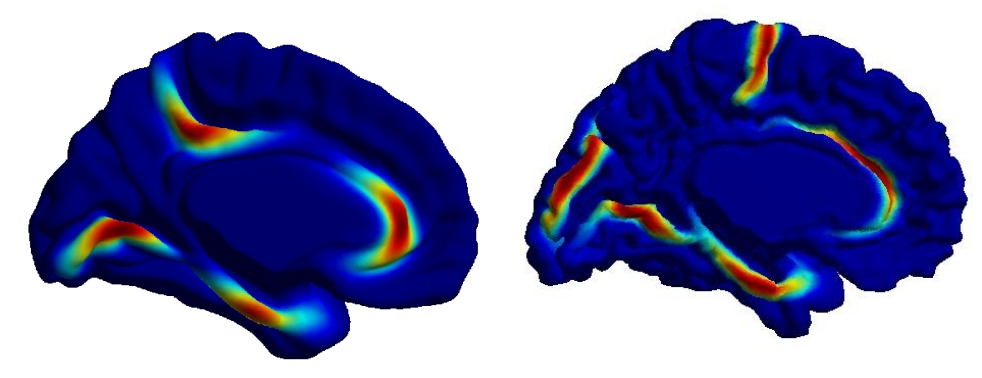

Fig. 1. We illustrate our anisotropic diffusion process on two examples, a template surface (left) and the mid-cortical surface of a subject (right). In both examples seed regions are placed and the color maps indicate how the diffusion follows the shape of folds.

The paper is organized as follows. We first describe a general setting to carry out tensor-based morphometry on surfaces, in Section 2.1. We then show how orientation estimates can be used to generate anisotropic filters on surfaces in 
Section 2.3. The method to rotate tensors according to neighboring fold orientation is developed in Section 2.4. Finally, in Section 3 we present experimental results on cross-sectional data that demonstrate that the anisotropic diffusion scheme is able to follow the orientation of neighboring folds, and that the method offers increased sensitivity in detecting shape differences in test studies. Using the proposed coherence-enhancing filters, we analyze how the shape of cortical folds is correlated with aging, in a database of 95 healthy subjects. We are able to find significant shape differences of the cortical surface associated with aging, which are strongly localized along specific folds. Such results would not be possible using isotropic diffusion kernels.

\section{Methods}

We first describe a general setting to carry out morphometric analysis on surfaces in Sections 2.1 and 2.2. We then develop anisotropic filters on surfaces in Section 2.3. Finally Section 2.4 combines these methods for estimating fold orientation.

We begin by introducing some notation. In this paper, all operations are performed on a template surface $\overline{\mathcal{M}}$ with a Riemannian metric $\mathrm{g}$. The surface $\overline{\mathcal{M}}$ represents the template shape of one hemisphere of the cerebral cortex. The Riemannian metric used is therefore the one induced by the imbedding in $\mathbb{R}^{3}$.

Bold face letters are used for vector fields and tensor fields, such as $\mathbf{u}, \mathbf{v} \in$ $\mathcal{T} \overline{\mathcal{M}}$, where $\mathcal{T} \overline{\mathcal{M}}$ is the tangent bundle of $\overline{\mathcal{M}}$. We denote the inner product according to the Riemannian metric as $\mathbf{g}(\mathbf{u}, \mathbf{v})$. In order to keep the notation simple, we use a global coordinate frame formed of two tangent vector fields, $\mathbf{x}_{1}, \mathbf{x}_{2} \in \mathcal{T} \overline{\mathcal{M}}$. We use lower indices for vector fields and upper indices for covector fields, such that $\mathbf{u}$ can be written in terms of the $\left[\mathbf{x}_{1}, \mathbf{x}_{2}\right]$ coordinate frame as

$$
\mathbf{u}=u^{1} \mathbf{x}_{1}+u^{2} \mathbf{x}_{2}
$$

Upper case letters are used for second order tensor fields (e.g.: T), with lower case letters such as $\mathbf{u}, \mathbf{v}$ for vector fields. The only exception to the upper/lower case convention for tensor fields is the Riemannian metric where $\mathbf{g}$ is used.

Using the $\left[\mathbf{x}_{1}, \mathbf{x}_{2}\right]$ frame, we write $\mathbf{g}$ the metric tensor expressed in this basis and $g_{i j}$, the $i, j$ component of the Riemannian metric. This allows us to define a point-wise dot-product between the tangent vector fields $\mathbf{u}, \mathbf{v}$ as

$$
\mathbf{g}(\mathbf{u}, \mathbf{v})=\left[u^{1}, u^{2}\right] \mathbf{g}\left[\begin{array}{c}
v^{1} \\
v^{2}
\end{array}\right]=\sum_{i, j \in\{1,2\}} g_{i j} u^{i} v^{j} .
$$

We use $\mathbf{g}^{-1}$ for the inverse of the metric tensor and $g^{i j}$ the $i, j$ component of this inverse. The $L^{2}$ norm of a second-order tensor field $\mathbf{T} \in \mathcal{T} \overline{\mathcal{M}} \times \mathcal{T} \overline{\mathcal{M}}$ is expressed as

$$
\|\mathbf{T}\|_{\mathbf{g}}^{2}=\int_{\overline{\mathcal{M}}}\|\mathbf{T}\|_{\mathbf{g}_{\mathbf{p}}}^{2} d \overline{\mathcal{M}}=\sum_{i, j, k, l \in\{1,2\}} \int_{\overline{\mathcal{M}}} g_{i k} g_{j l} T^{i j} T^{k l} d \overline{\mathcal{M}}
$$


where the notation $\|\mathbf{T}\|_{\mathbf{g}_{\mathrm{p}}}^{2}$ represents the $l^{2}$ norm at a point $\mathbf{p} \in \overline{\mathcal{M}}$. Where it is convenient to do so, we will denote a multiplication between tensors, say $\mathbf{T}, \mathbf{S}$, by writing the tensors next two each other and including a metric term if necessary. For example, a multiplication between two tensors is given by

$$
\mathbf{T g S}=\sum_{j, k \in\{1,2\}} T^{i j} g_{j k} S^{k l} .
$$

\subsection{Morphometric Analysis on Surfaces}

We now review the framework of tensor-based morphometry used for shape analysis [9]. The shape of each hemisphere of the human cerebral cortex is represented as a single surface embedded in Euclidean space $\mathbb{R}^{3}$. Let $\mathcal{M}_{s} \subset \mathbb{R}^{3}, k=1, \ldots, N$ be surfaces representing the cerebral cortices of $N$ individuals, one surface per hemisphere. We suppose that these surfaces are homeomorphic to a sphere.

The surfaces are mapped onto a common template $\overline{\mathcal{M}}$ through a process known as surface registration [7]. Surface registration produces a diffeomorphic map $\psi_{s}: \overline{\mathcal{M}} \rightarrow \mathcal{M}_{s}$ that matches the features of $\overline{\mathcal{M}}$ onto the corresponding features on $\mathcal{M}_{s}$. The amount of deformation required to map $\overline{\mathcal{M}}$ to $\mathcal{M}_{s}$ is used to analyze the shape of individual surfaces. This amount is computable from the diffeomorphic map $\psi_{s}$. We obtain a tensor [9] representing the amount of deformation required to morph $\mathcal{M}_{s}$ into $\overline{\mathcal{M}}$ as

$$
\mathbf{T}_{s}=\left[\operatorname{grad}\left(\psi_{s}\right)\right]^{t}\left[\operatorname{grad}\left(\psi_{s}\right)\right] .
$$

In this case, $\mathbf{T}_{s}$ forms a $2 \times 2$ positive symmetric definite tensor field on the surface. A multivariate statistical test is then applied on the tensor field $\mathbf{T}_{s}$ in order to see if there is any significant correlation with an exterior factor. The method for statistical analysis is described in $[6,9]$ and we provide an example result in Fig. 5. Unless we refer to a specific subject, the ${ }_{s}$ index will be dropped and we will use the notation $\mathbf{T}$ for a tensor field on $\overline{\mathcal{M}}$ in the rest of this paper.

\subsection{Diffusion of Tensor Fields}

In this section, we present a method for diffusion of tensor fields on surfaces. The tensor laplacian, in terms of the vector fields $\mathbf{x}_{1}, \mathbf{x}_{2}$ is given as

$$
\Delta \mathbf{T}=\sum_{i, j \in\{1,2\}} \nabla_{\mathbf{x}_{i}} g^{i j} \nabla_{\mathbf{x}_{j}} \mathbf{T},
$$

where $\nabla$ is the covariant derivative. Equation 6 allows to perform diffusion on surfaces as

$$
\frac{\partial \mathbf{T}}{\partial t}=\Delta \mathbf{T} .
$$




\subsection{Anisotropic Diffusion of Scalar Fields along Folds}

The anisotropic filter for surface shape analysis we propose is designed to follow the orientation of neighboring crests and troughs, as shown in Figure 1. The general anisotropic diffusion equation used to build these filters for a scalar field $f: \bar{M} \rightarrow \mathbb{R}$ is given as [10]:

$$
\frac{\partial f}{\partial t}=\sum_{i, j, k \in\{1,2\}} \frac{1}{\sqrt{|\mathbf{g}|}} \partial_{\mathbf{x}_{i}} \sqrt{|\mathbf{g}|} A^{i}{ }_{j} g^{j k} \partial_{\mathbf{x}_{k}} f,
$$

where $\mathbf{A}: \mathcal{T} \overline{\mathcal{M}} \rightarrow \mathcal{T} \overline{\mathcal{M}}$ is a symmetric positive definite operator which controls diffusion speed on $\overline{\mathcal{M}}$, and $|\mathbf{g}|$ is the determinant of $\mathbf{g}$.

The most direct method by which $\mathbf{A}$ can be adapted to follow curve patterns on $\overline{\mathcal{M}}$ is to estimate the local orientation of the folding pattern. Let $\mathbf{v}_{\theta}$ be an estimate of the local fold orientation $\left(\left\|\mathbf{v}_{\theta}\right\|_{\mathbf{g}_{\mathrm{p}}}=1\right)$ on $\overline{\mathcal{M}}$. Then $\mathbf{A}$ can be chosen as

$$
\mathbf{A}=\alpha \mathbf{I}+(1-\alpha)\left(\left(\mathbf{v}_{\theta} \otimes \mathbf{v}_{\theta}\right) \mathbf{g}\right),
$$

where $\alpha \in] 0,1]$ is the anisotropic factor, $\mathbf{I}$ is the identity operator, $\otimes$ is a tensor product. The challenge is to select $\mathbf{v}_{\theta}$ such that it is a good estimate of the local folding pattern orientation.

The principal curvature directions on a surface provide a first-hand estimate of the local orientation of its folds. The curvature of the surface is low in the direction parallel to the fold, and is high perpendicular to fold orientation. The principal curvatures are measured by the shape operator. Let $\mathbf{n}$ be the unit surface normal. The shape operator $\mathbf{C}$ of a surface is defined as the covariant derivative of the normal $\mathbf{n}$ along $\mathbf{u}$ as

$$
\mathbf{C u}=-\nabla_{\mathbf{u}} \mathbf{n},
$$

where $\nabla$ is the covariant derivative in $\mathbb{R}^{3}$. The covariant derivative of the normal is a tangent vector field on $\overline{\mathcal{M}}$. To focus on the magnitude of the principal curvatures, we use the absolute value of the curvature. Let, the following definition of $\mathbf{S}$ be given as the non-negative symmetric tensor such that, if $\mathbf{v}_{1}, \mathbf{v}_{2}$ are the two eigenvectors of $\mathbf{C}$ with eigenvalue $\lambda_{1}, \lambda_{2}$. Then, $\mathbf{S}$ is defined as

$$
\mathbf{S}=\left|\lambda_{1}\right| \mathbf{v}_{1} \otimes \mathbf{v}_{1}+\left|\lambda_{2}\right| \mathbf{v}_{2} \otimes \mathbf{v}_{2} .
$$

In other words, the tensor $\mathbf{S}$ is the tensor whose eigenvalues are $\left|\lambda_{1}\right|,\left|\lambda_{2}\right|$. This allows us to define an estimator of the local orientation $\mathbf{v}_{\theta}$ of neighboring folds as the eigenvector of $\mathbf{S}$ with smallest eigenvalue, i.e.

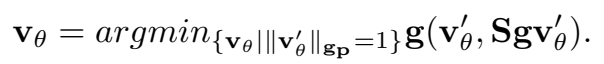

However, between creases where curvature is small, the principal directions of $\mathbf{S}$ may not accurately reflect neighboring fold directions. Hence, we first apply a small amount of regularization to the tensor $\mathbf{S}$ using tensor diffusion as expressed by Equation 7. Let, $\mathbf{S}(t)$ be the result of applying diffusion on $\mathbf{S}$ for a time $t$ 


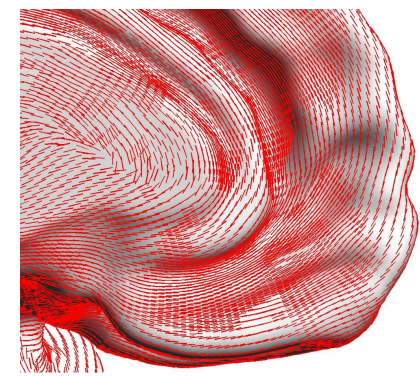

(a) Generation of a vector field $\mathbf{v}_{\theta}$ using the normal curvature of neighboring folds. The vector field shown is parallel to the folds on the patch.

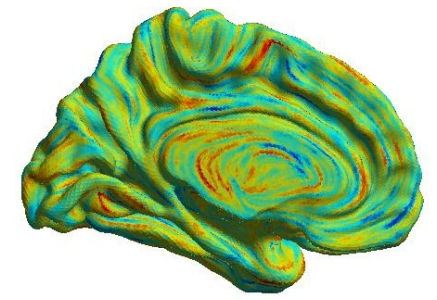

(b) Medial view of the Left Hemisphere

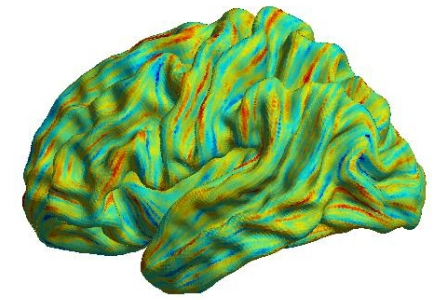

(c) Exterior view of the Left Hemisphere

Fig. 2. A visualization of orientation fields on the template surface using line integral convolution. In a qualitative sense, the vector field shown follows the major folds on the cortical surface.

according to Equation 7. We then apply Equation 12 to obtain the fold direction that is perpendicular to the direction of highest curvature.

Typically, we chose an anisotropic factor $\alpha$ of 0.1 for diffusion and a diffusion time of 100 to estimate local orientation. A few results of scalar field diffusion are shown in Figure 1 and Figure 2(a). It should be noted that the matrix field $\mathbf{A}$ defined in this section will contain discontinuities, corresponding to the discontinuities of the vector field $\mathbf{v}_{\theta}$. In order for the anisotropic diffusion process to be well defined, a very small amount of regularization is also applied to $\mathbf{A}$, again by using isotropic diffusion (Equation 7).

\subsection{Anisotropic Diffusion of Tensor Fields on Surfaces}

We now consider diffusion of tensor fields on surfaces. Recall that Equation 7 provides a diffusion process for tensors. The center column of Figure 3 illustrates that the basic diffusion equation provided in Equation 7 does not result in a diffusion scheme that follows local fold orientation. This is a problem for our application where we intend to study shape differences that affect fold shape. In particular, we wish to align the diffusion process using the orientation of neighboring folds. 
To see how tensors can be realigned according to neighboring fold orientation, consider the following local rotation process. Let $\mathbf{R}_{\theta}$ be a rotation operator, such that it preserves the local orientation on surfaces and does not change the inner product between two vector fields, i.e. $\mathbf{g}\left(\mathbf{R}_{\theta} \mathbf{u}, \mathbf{R}_{\theta} \mathbf{v}\right)=\mathbf{g}(\mathbf{u}, \mathbf{v})$. Using matrix notation, a rotated frame $\mathbf{t}_{1}, \mathbf{t}_{2}$ can be written in term of the original frame as

$$
\left[\begin{array}{l}
\mathbf{t}_{1} \\
\mathbf{t}_{2}
\end{array}\right]=\mathbf{R}_{\theta}\left[\begin{array}{l}
\mathbf{x}_{1} \\
\mathbf{x}_{2}
\end{array}\right]=\mathbf{g}^{-\frac{1}{2}}\left[\begin{array}{cc}
\cos (\theta) & -\sin (\theta) \\
\sin (\theta) & \cos (\theta)
\end{array}\right] \mathbf{g}^{\frac{1}{2}}\left[\begin{array}{l}
\mathbf{x}_{1} \\
\mathbf{x}_{2}
\end{array}\right]
$$

The technique that we propose in this paper is to compute an optimal rotation operator $\mathbf{R}_{\theta}$ such that it best preserves the tensor field $\mathbf{S}$. Then, the same rotation operator is applied to the deformation tensors computed in Equation 5.

We will now define a diffusion scheme for tensors in a rotated coordinate system. Consider a rotated differential operator as

$$
\begin{aligned}
\mathbf{R}_{\theta}^{-1} \nabla_{\mathbf{x}_{k}}\left(\mathbf{R}_{\theta} \mathbf{S R}_{\theta}^{t}\right) \mathbf{R}_{\theta}^{t-1} & =\nabla_{\mathbf{x}_{k}} \mathbf{S}+v_{k}^{\theta}\left(\mathbf{g}^{-\frac{1}{2}}\left[\begin{array}{cc}
0 & 1 \\
-1 & 0
\end{array}\right] \mathbf{g}^{\frac{1}{2}} \mathbf{S}+\mathbf{S g}^{\frac{1}{2}}\left[\begin{array}{cc}
0 & -1 \\
1 & 0
\end{array}\right] \mathbf{g}^{-\frac{1}{2}}\right) \\
& =\nabla_{\mathbf{x}_{k}} \mathbf{S}+v_{k}^{\theta} D \mathbf{S},
\end{aligned}
$$

where $D \mathbf{S}$ is defined as the term in parentheses and $v_{k}^{\theta}$ designates a parameter that needs to be estimated. Equation 14 allows us to define a rotated anisotropic diffusion scheme for tensors as

$$
\frac{\partial \mathbf{S}}{\partial t}=\sum_{i, j, k \in\{1,2\}} \nabla_{\mathbf{x}_{i}} A^{i}{ }_{j} g^{j k}\left(\nabla_{\mathbf{x}_{k}} \mathbf{S}+v_{k}^{\theta} D \mathbf{S}\right) .
$$
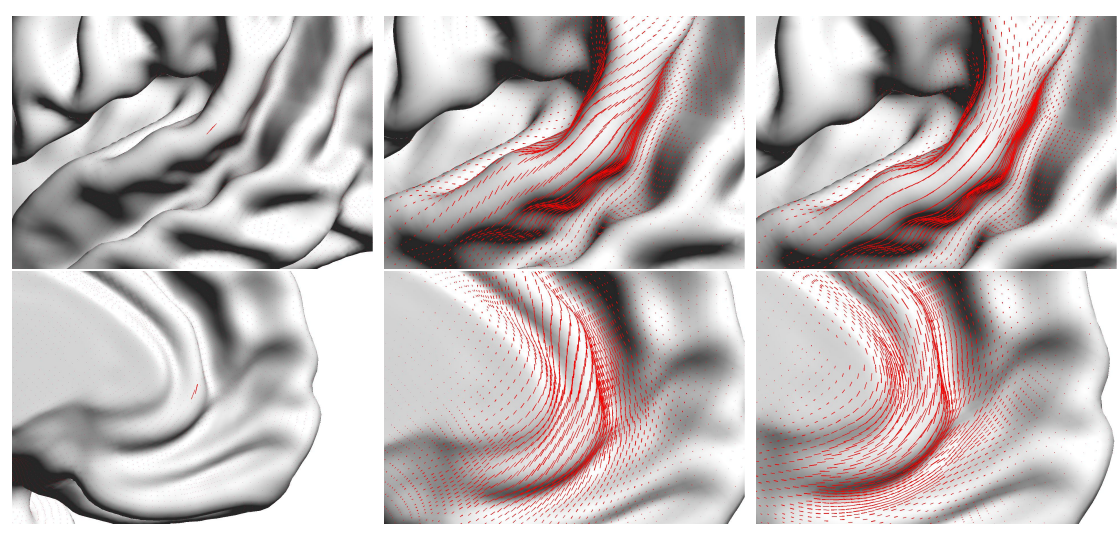

Fig. 3. An illustration of aligning tensors with neighboring folds while performing diffusion. An anisotropic second-order tensor (shown on the left) was diffused on a template surface (top: left superior temporal gyros, bottom: left rostral cingulate gyrus). The center needle map shows the direction of the highest eigenvalue of the diffused tensor. The diffusion results in a tensor field that does not maintain a constant orientation with nearby geometric folds. The right needle map shows how the method presented in Section 2.4 causes the diffused tensors to be aligned with nearby folds. 
Let $\mathbf{w}=\left[w_{1}, w_{2}\right]$ be an estimator for $v_{k}^{\theta}$. We optimize the value of $\mathbf{w}$, by minimizing the norm of the differential of $\mathbf{S}$ over the entire diffusion process as

$$
w_{k}=\operatorname{argmin}_{w_{k}^{\prime}} \int_{[0, t]}\left\|\partial_{\mathbf{x}_{k}} \mathbf{S}(z)+D \mathbf{S}(z) w_{k}^{\prime}\right\|_{\mathbf{g}_{\mathbf{p}}}^{2} d z .
$$

Using calculus of variations, we obtain that the solution $\mathbf{w}$ of Equation 16 is

$$
w_{k} \int_{[0, t]}\|D \mathbf{S}(z)\|_{\mathbf{g}_{\mathbf{p}}}^{2} d z=-\int_{[0, t]} \operatorname{trace}\left(\partial_{\mathbf{x}_{k}} \mathbf{S}(z) \mathbf{g} D \mathbf{S}(z) \mathbf{g}\right) d z,
$$

where trace is the trace of the tensor. An illustration of performing diffusion while rotating tensors according to Equation 17 is provided in Figure 3. The left column shows a tensor field which is equal to zero everywhere, except at one location, where it equals $\mathbf{v}_{\theta} \otimes \mathbf{v}_{\theta}$. The result of diffusing this tensor field without computing an optimal rotation field is shown in the center column and the result of diffusion with the optimal rotation field is shown in the right column. The rotation causes the diffused tensor field to follow the orientation of neighboring folds.

\section{$3 \quad$ Experimental Results}

We now provide additional illustrations and promising applications of the proposed diffusion process. We begin by evaluating the method on the cortical surfaces of individual subjects. Here our goal is to select a few major folds on the cortical surface (such as the central sulcus, hippocampal gyrus and superior temporal sulcus), and to determine if the anisotropic diffusion process follows these folds. Each fold was first manually identified on the template surface $\overline{\mathcal{M}}$. Then a geodesic distance function was used to measure the distance to the fold on the template surface, as shown in Figure 4(a). The level sets of the geodesic distance function provide an approximation to the direction the diffusion should follow in order to remain parallel to the segmented fold.

We used surface registration to map the geodesic distance functions computed on the template surface onto 15 cortical surfaces (left hemispheres) of individual subjects. Let $\bar{\rho}$ be the geodesic distance function on the template surface and $\rho=\bar{\rho}\left(\psi^{-1}\right)$ the mapped geodesic distance function on a specific subject (see Fig. 4(a)). Let $e^{t \Delta_{\alpha}}$ be the solution to the anisotropic diffusion given in Equation 8 with $\alpha=0.1$. We first computed a diffused value for the geodesic distance function as $\mu=e^{t \Delta_{\alpha}} \rho$, where $e^{t \Delta_{\alpha}}$ is the result of applying diffusion for a time $t$ according to Equation 8. Then, we determined the extent to which diffusion follows level sets of the geodesic distance function by measuring how it varies compared to $\mu$ as

$$
E(\rho)=\sqrt{e^{t \Delta_{\alpha}}(\rho-\mu)^{2}} .
$$

In the event where diffusion perfectly follows the level set curves of $\rho, E(\rho)$ is almost zero. We computed the diffusion until the kernel covered a region of about 


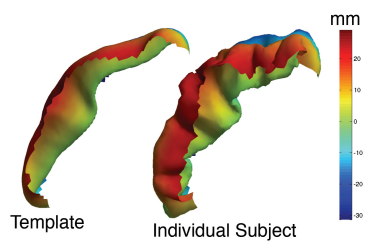

(a) Level-set on the central sulcus. Left: $\bar{\rho}$, right: $\rho$

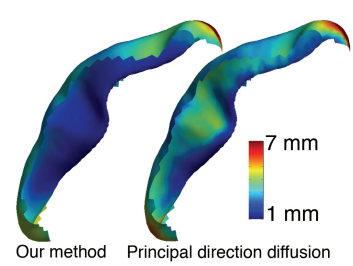

(d) Central Sulcus: Average of $E(\rho)$ over 15 subjects

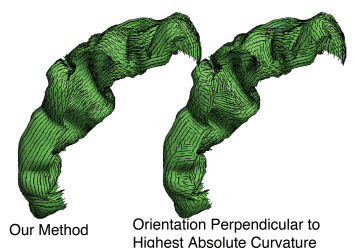

(b) Orientation field using principal curvature and our method

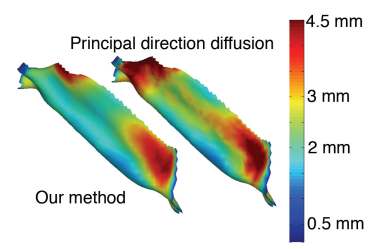

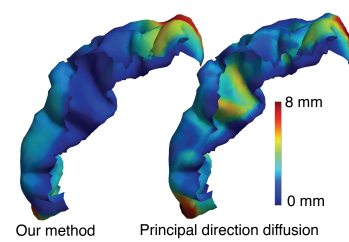

(c) Central Sulcus: Result of $E(\rho)$ on an individual surface

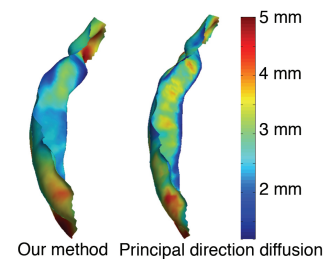

(e) Hippocampal Gyrus: (f) Temporal Sulcus: AverAverage of $E(\rho)$ over 15 age of $E(\rho)$ over 15 subjects subjects

Fig. 4. Anisotropic diffusion is able to follow the level sets of a geodesic distance function to a segmented fold on individual cortical surfaces, as measured by $E(\rho)$. See the text for a discussion.

$4 \mathrm{~cm}$ on the cerebral cortex along the longest axis. We display the result for the three sulci in the second row of Figure 4. As a point of comparison, we used an anisotropic diffusion scheme where the diffusion was constrained to the direction of the smaller principal curvature, as measured by $\mathbf{S}(0)$ (see the example on the left of Figure 4(b)). The result of this anisotropic kernel is shown in Figure 4 under the label "principal direction diffusion" .

In the second row of Figure 4, we show that the value of $E(\rho)$ is much smaller on average in our method as compared with principal direction diffusion. The orientation field produced by our method is more parallel to the major fold and is not influenced by the small changes in orientation along the sulcal wall, as opposed to that obtained using principal direction diffusion (see Fig. 4(b)). Also, most of the failure cases are due to the fact that the geodesic distance function is based only on the segmented fold, whereas our method captures neighboring fold orientation as well.

In a second experiment we used anisotropic diffusion to reveal significant shape differences in the left hemisphere within a population of 95 adults aged 19 to 85 years old, with a median age of 44 years, selected from the ICBM database. For illustration purposes, only the left hemisphere was used. We used multivariate tensor-based $T$-statistics on this population to compare anisotropic and isotropic diffusion [6]. 


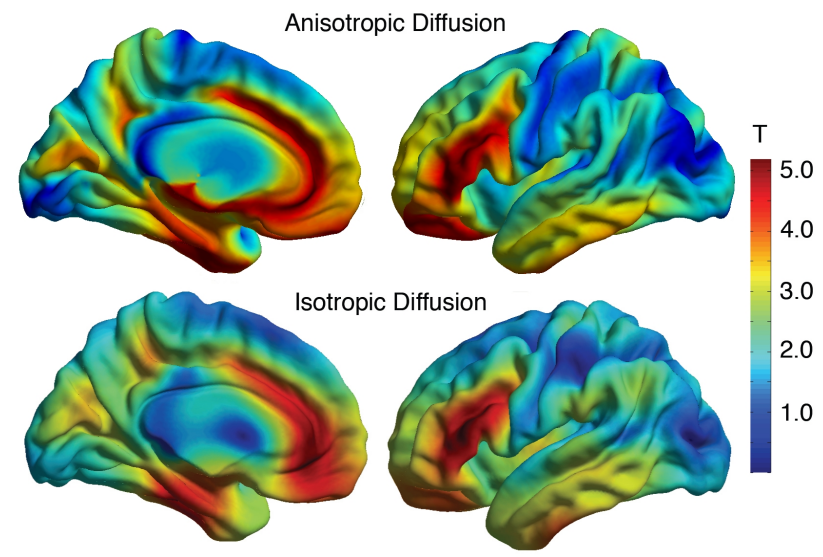

Fig. 5. Multivariate tensor statistics using isotropic and anisotropic diffusion. Anisotropic diffusion shows peak regions that are well localized around specific folds, whereas peaks for isotropic diffusion spread across multiple folds.

To compute regression statistics, first a tensor field for each subjects was computed according to Equation 5. Then, each tensor field was diffused according to Equation 15 with parameters $\alpha=0.1$ and for a time $t=400\left(\mathrm{~mm}^{2}\right)$. Let $\hat{\mathbf{T}}_{s}$ be the resulting tensor field after diffusion. The following linear model was used to compute the regression with age and gender:

$$
\log \left(\hat{\mathbf{T}}_{s}\right)=\hat{\mathbf{M}}+\hat{\mathbf{T}}_{a g e} \text { age }_{s}+\hat{\mathbf{T}}_{\text {gender }} \text { gender }_{s}+\mathbf{E}_{s}
$$

where $\log$ is the tensor logarithm using the Log-Euclidean model of [1], age and gender $_{s}$ are the age and gender of subject $s$, and $\mathbf{E}_{s}$ is the residual error of the linear model. A least-squares fit was used to find the regression tensor field corresponding to the mean $\hat{\mathbf{M}}$, age $\hat{\mathbf{T}}_{\text {age }}$ and gender $\hat{\mathbf{T}}_{\text {gender }}$. A multivariate $T$ test for age correlation was then computed at each vertex using SurfStat [11].

We first provide a comparison between isotropic diffusion and anisotropic diffusion in Figure 5 in terms of the resulting $T$-test. Although a fair comparison is difficult because there are many diffusion parameters to be selected, Figure 5 demonstrates that results using anisotropic diffusion are well localized around specific folds. By this, we mean that regions where $T$ is high do not spread over multiple folds with anisotropic diffusion whereas regions where $T$ is high with isotropic diffusion spread across several folds and also produce lower peak values for $T$.

We computed thresholds for significance to control for false positives using a permutation test with 10000 permutations. The threshold for significance $(p<0.05)$ using multivariate tensor-based morphometry was $|T|>4.752$. We display significance results in Figure 6. These results indeed show that anisotropic diffusion is able to find regions of significant atrophy localized to a single fold. 


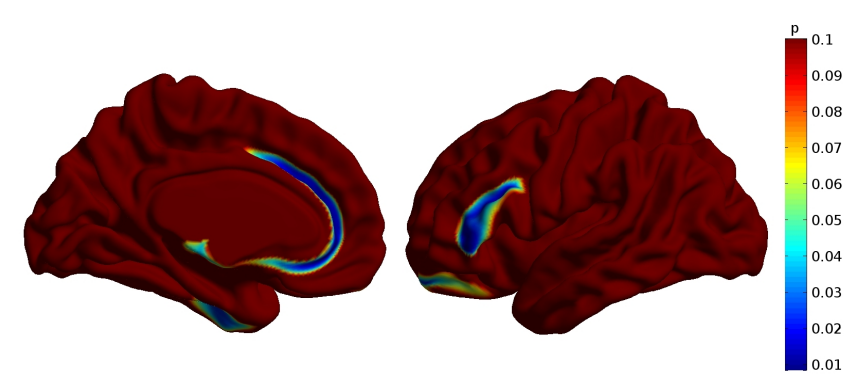

Fig. 6. Probability maps of multivariate tensor statistics using anisotropic diffusion. Statistically significant regions are well localized around specific folds.

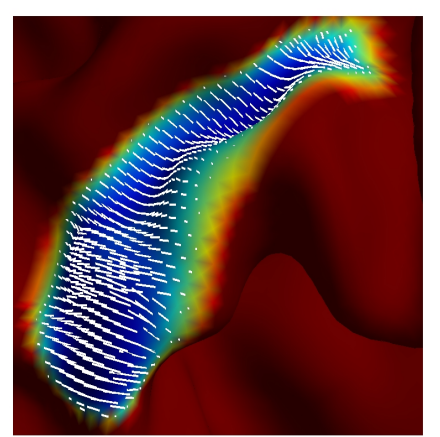

Left Frontal Lobe

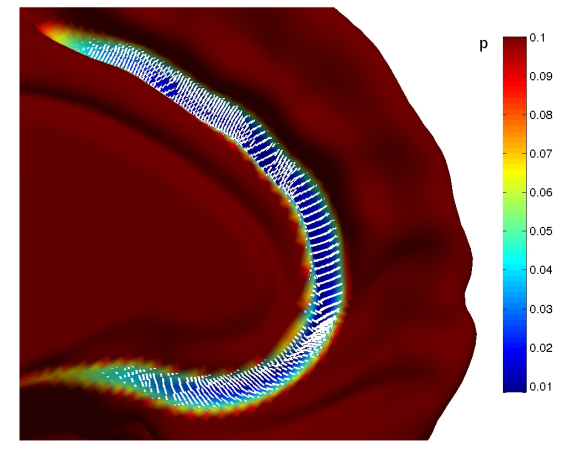

Left Cingulate Sulcus

Fig. 7. A close up view of the maximum negative correlation with aging in the Cingulate sulcus and Frontal lobe. The white lines show the direction which indicates maximum negative correlation associated with aging.

We also tried to determine which direction showed the highest correlation with the subject's age and wether this correlation was positive or negative. We extracted from the tensor fields $\hat{\mathbf{T}}_{a g e}$, the orientation which showed the highest correlation with age:

$$
\mathbf{v}_{\max }=\operatorname{argmax}_{\left\{\mathbf{v}^{\prime} \mid\left\|\mathbf{v}^{\prime}\right\|_{\mathbf{g}_{\mathbf{p}}}=1\right\}}\left|\mathbf{g}\left(\mathbf{v}^{\prime}, \hat{\mathbf{T}}_{a g e} \mathbf{g} \mathbf{v}^{\prime}\right)\right|
$$

This orientation field of maximum correlation is shown in Figure 7 and corresponds to a negative correlation in both the cingulate sulcus and a region of the frontal lobe. The orientation field shows that the folds in question have a smaller width in the elderly populations. We tested the significance of the shape difference along this direction using the permutation test presented in [3] and found the result to be slightly more significant than presented in Figure 6 . 


\section{Conclusion}

This paper proposes a new method for morphometric studies of cortical surfaces.

The proposed anisotropic tensor diffusion process on surfaces can be used to highlight deformations that affect sulcal folds by enhancing deformation maps along them. Using the proposed coherence-enhancing filters, we are able to find anisotropic shape differences of the cortical surface associated with aging that are well localized along specific folds.

\section{Acknowledgments}

This work was supported by grants from FQRNT and NSERC.

\section{References}

1. Arsigny, V., Fillard, P., Pennec, X., Ayache, N.: Log-Euclidean metrics for fast and simple calculus on diffusion tensors. Magnetic resonance in medicine 56(2), 411-421 (2006)

2. Boucher, M., Evans, A., Siddiqi, K.: Oriented morphometry of folds on surfaces. In: Information Processing in Medical Imaging. pp. 614-625. Springer (2009)

3. Boucher, M., Evans, A., Siddiqi, K.: A Texture Manifold for Curve-Based Morphometry of the Cerebral Cortex. Medical Computer Vision. Recognition Techniques and Applications in Medical Imaging pp. 174-183 (2011)

4. Chung, M., Worsley, K., Evans, A.: Tensor-based brain surface modeling and analysis. Computer Vision and Pattern Recognition, 2003. Proceedings. 2003 IEEE Computer Society Conference on 1, 467-473 (2003)

5. Desbrun, M., Meyer, M., Schröder, P., Barr, A.: Implicit fairing of irregular meshes using diffusion and curvature flow. Proceedings of the 26th annual conference on Computer graphics and interactive techniques pp. 317-324 (1999)

6. Fillard, P., Arsigny, V., Ayache, N., Pennec, X.: A Riemannian Framework for the Processing of Tensor-Valued Images. Lecture Notes in Computer Science 3753, 112 (2005)

7. Fischl, Sereno, Dale: Cortical surface-based analysis. II: Inflation, flattening, and a surface-based coordinate system. Neuroimage 9(2), 195-207 (1999)

8. Franken, E., Duits, R.: Crossing-preserving coherence-enhancing diffusion on invertible orientation scores. IJCV 85(3), 253-278 (2009)

9. Lepore, N., Brun, C.A., Chou, Y.Y., Chiang, M.C., Dutton, R.A., Hayashi, K.M., Luders, E., Lopez, O.L., Aizenstein, H., Toga, A.W., Becker, J.T., Thompson, P.M.: Generalized Tensor-Based Morphometry of HIV/AIDS Using Multivariate Statistics on Deformation Tensors. Medical Imaging, IEEE Transactions on 27(1), 129-141 (2008)

10. Weickert, J.: Anisotropic diffusion in image processing, vol. 256 (1998)

11. Worsley, K., Taylor, J., Carbonell, F., Chung, M., Duerden, E., Bernhardt, B., Lyttelton, O., Boucher, M., Evans, A.: SurfStat: A Matlab toolbox for the statistical analysis of univariate and multivariate surface and volumetric data using linear mixed effects models and random field theory. NeuroImage 47, S102-S102 (2009) 